The Astrophysical Journal, 163:375-382, 1971 January 15

(C) 1971 The University of Chicago All rights reserved Printed in U S A

\title{
TEMPERATURE VARIATION AND THE SOLAR OBLATENESS*
}

\author{
ANDREW P. INGERSOLL \\ Division of Geological Sciences, California Institute of Technology, and Hale Observatories, \\ Carnegie Institute of Washington, California Institute of Technology
}

AND

EDward A. Spiegel

Department of Astronomy, Columbia University

Received 1970 September 21; revised 1970 October 8

\section{ABSTRACT}

Dicke and Goldenberg's oblateness measurement may be explained by an equatorial temperature excess of $30^{\circ} \mathrm{K}$, smoothly distributed in optical depths $\leq 001$ The resulting brightness variation with solar latitude is concentrated close to the limb, and it is not possible, with data presently available, to distinguish such variation from true oblateness

\section{INTRODUCTION}

In a widely discussed series of observations, Dicke and Goldenberg (1967) attempted to determine the solar oblateness by measuring the variation with latitude of the flux from the outer portion of the solar disk. They projected an image of the Sun on a circular occulting disk and measured the flux in narrow sectors extending from the occulting disk outward beyond the limb. The part of this flux which varied with latitude was the "signal" used to infer the solar oblateness. They concluded that the observed signal indicated an oblateness of $5 \times 10^{-5}$, about 5 times the value expected from the observed surface rotation of the Sun. Possible causes of such an oblateness have been debated, and Dicke $(1970 a, b)$ has recently reviewed the various suggestions.

It is also possible that the observed signal results from a variation of brightness with solar latitude (Durney and Roxburgh 1969). In this case the signal amplitude should depend on the amount of the solar image exposed beyond the occulting disk. This amount is characterized by the radial angular distance $\delta$ from the edge of the occulting disk to the mean solar limb. In the observations three values of $\delta$ were used $\left(6 " .5,12^{\prime \prime} .9,19 \prime \prime 1\right)$, corresponding to three values of the radius of the occulting disk. ${ }^{1}$ If the signal were the result of true oblateness, the amplitude would be substantially independent of $\delta$. Dicke and Goldenberg assumed that if the signal were the result of brightness variation, the amplitude would be proportional to $\delta$. But as we shall see, if a latitude variation of temperature occurs at sufficient height in the solar atmosphere, the resulting signal amplitude is proportional to $\delta^{1 / 2}$. Such a dependence on $\delta$ is not excluded by the published data, and the suggested value of the solar oblateness is open to question.

\section{DEPENDENCE ON $\delta$}

In this discussion we shall use a spherical coordinate system $(r, \theta, \phi)$ centered on the Sun, with the Earth at $\theta=0$. We neglect the finite angular size of the Sun; hence all rays to the Earth are parallel to the polar coordinate axis. For such rays let $\mu=|\cos \theta|$.

* This work has been supported by the National Science Foundation and by the National Aeronautics and Space Administration

1 Professor Dicke has pointed out that these distances are defined in terms of the light fluxes measured in the experiments A particular light flux curve is assumed (Dicke 1970a), but because of poor "seeing" the actual light flux curve is not the theoretical one Thus the values of $\delta$ are somewhat uncertain, but this uncertainty is probably not important in the discussion which follows 
We consider here a model in which the surfaces of constant optical depth $\tau$ are spherical. The model has no oblateness in the sense used by Dicke and Goldenberg, and the "signal" it produces results from a variation of brightness (i.e., temperature) with latitude. This signal will be compared with that part of the observed signal which is not associated with the surface rotation. Later we shall argue that the implied variation in temperature is sufficiently small that the resulting difference between surfaces of constant $\tau$ and of constant gravitational potential is negligible. We also neglect the slight range in solar latitude traversed by a ray to the Earth.

Consider now the radiation from a volume element $r^{2} \sin \theta d r d \theta d \phi$ at optical depth $\tau(r)$. Let the optical distance to the Earth through the intervening layers of the Sun be denoted by $\tau_{+}(\mu, \tau)$ or $\tau_{-}(\mu, \tau)$, depending on whether $\theta$ is less than or greater than $\frac{1}{2} \pi$. The fraction transmitted to the Earth is $e^{-\tau_{ \pm}}$, where $\tau_{ \pm}$is either $\tau_{+}$or $\tau_{-}$, whichever is appropriate. Then the contribution of this volume element to the power per unit solid angle reaching the Earth is

$$
k \rho B e^{-\tau_{ \pm}} \boldsymbol{r}^{2} \sin \theta d r d \theta d \phi=r^{2} B e^{-\tau} \pm d \tau d \mu d \phi,
$$

where $k$ is the mass absorption coefficient, $\rho$ is the density, and $B$ is the integrated Planck function. Let $r_{0}$ be the radius of the occulting disk when it is projected onto the solar disk. A ray to the Earth from the point $(r, \theta, \phi)$ will pass outside the occulting disk provided $\mu \leq \mu_{0}$, where

$$
\mu_{0}(r)=\left[1-\left(\frac{r_{0}}{r}\right)^{2}\right]^{1 / 2} \approx\left[\frac{2\left(r-r_{0}\right)}{r_{0}}\right]^{1 / 2} .
$$

Then the power per unit solid angle received from a sector $\phi_{1} \leq \phi \leq \phi_{2}$ is the integral of expression (1) over $\mu$ from 0 to $\mu_{0}$, over $\phi$ from $\phi_{1}$ to $\phi_{2}$, and over $\tau$ from zero to infinity. (We shall see later that $\tau / \mu_{0} \gg 1$ is equivalent to $\tau \rightarrow \infty$.) The difference of this integral from equator to pole is the observed signal. In the same units the signal amplitude per unit of $\phi$ is

$$
A=\int_{0}^{\infty} r^{2} \Delta B d \tau \int_{0}^{\mu_{0}}\left(e^{-\tau_{+}}+e^{-\tau_{-}}\right) d \mu \equiv A_{+}+A_{-},
$$

where $\Delta B(\tau)$ is $B(\tau$, equator $)-B(\tau$, pole), and the near- and far-side contributions are $A_{+}$and $A_{-}$, respectively.

To compute $\tau_{ \pm}$we need a model relating the running variables $\tau^{\prime}, \mu^{\prime}, r^{\prime}$, evaluated along a ray to the Earth, to the fixed variables $\tau, \mu, r$, evaluated at the endpoint of the ray. We consider a particular model in which

$$
\tau^{\prime}=\tau \exp \left[-\left(r^{\prime}-r\right) / \lambda\right]
$$

A reasonable representation of $\tau^{\prime}$ in the upper photosphere and lower chromosphere is obtained with $\lambda=60 \mathrm{~km}$ (Dicke 1970a). Then the optical distance from a point $(r, \theta, \phi)$ on the near side of the Sun is

$$
\tau_{+}(r, \mu)=\int_{0}^{\tau} \frac{d \tau^{\prime}}{\mu^{\prime}}=\tau \int_{0}^{\infty} \frac{e^{-t}}{\mu^{\prime}} d t
$$

where $t=\left(r^{\prime}-r\right) / \lambda$. Along the ray, $\mu^{\prime}$ varies as

where

$$
\mu^{\prime}=\left[1-\frac{1-\mu^{2}}{(1+t \lambda / r)^{2}}\right]^{1 / 2} \approx \mu\left(1+t / x^{2}\right)^{1 / 2}
$$

$$
x^{2}=\frac{\mu^{2}}{\left(1-\mu^{2}\right)} \frac{r}{2 \lambda} \text {. }
$$


The approximation made in equation (6) fails when $t$ approaches the value $r / \lambda$, but the contribution from this part of the ray path to the integral (5) is negligible.

On introducing equation (6) into the integral (5), we find

$$
\tau_{+}=\sqrt{ } \pi \frac{\tau x}{\mu} e^{x^{2}}[1-\operatorname{erf}(x)]
$$

whence

where

$$
\begin{array}{ll}
\tau_{+} \sim \frac{\tau}{\mu}, & x \gg 1, \\
\tau_{+} \sim \tau \beta, & x \ll 1,
\end{array}
$$

and for $r \sim R=$ the solar radius, $\beta \sim 2 \times 10^{2}$. In obtaining equation (9b) we must note that small $x$ implies small $\mu$. The limit (9a) is the usual plane-parallel approximation, and the limit $(9 b)$ was given by Dicke $(1970 a)$.

A similar calculation yields

$$
\tau_{-}(r, \mu)=2 \tau \beta \exp \left\{\frac{r}{\lambda}\left[1-\left(1-\mu^{2}\right)^{1 / 2}\right]\right\}-\tau_{+}(r, \mu) .
$$

For $|r-R| \ll R$ the important contributions from the far side of the Sun originate in regions where $\mu \ll 1$, in which case expression (11) reduces to

$$
\tau_{-}=\sqrt{ } \pi \frac{\tau x}{\mu} e^{x^{2}}[1+\operatorname{erf}(x)],
$$

where here $x^{2} \approx \frac{1}{2} \mu^{2} r / \lambda$. The extremes of $\tau_{-}$corresponding to those of $\tau_{+}$are

$$
\begin{array}{rlrl}
\tau_{-} & \sim 2 \tau \beta e^{x^{2}}, & & x \gg 1, \\
\tau_{-} \sim \tau \beta, & & x \ll 1 .
\end{array}
$$

Consider first $A_{+}$, the signal from the near side as defined in equation (3). The integral in this definition runs from 0 to $\mu_{0}$ where $\mu_{0}$ is approximately $0.12,0.16$, and 0.20 for the three values of $\delta$ used in the observations. Equation (7) then indicates that the corresponding values of $x$ at $\mu=\mu_{0}$ are about 12,16, and 20. From equations (9) we see that the plane-parallel approximation holds over most of the range of integration of $\mu$, and breaks down only when $\mu$ is close to zero. In the neighborhood of small $\mu$ the integrand $\exp \left(-\tau_{+}\right)$has its smallest value, and the contribution from this region amounts to less than 10 percent of the whole for the three $\mu_{0}$ considered. It is therefore reasonable to use equation (9a) for $\tau_{+}$, and we find

$$
\int_{0}^{\mu_{0}} e^{-\tau+}+d \mu=\mu_{0} E_{2}\left(\tau / \mu_{0}\right)
$$

where $E_{2}$ is the second exponential integral.

We may estimate the magnitude of the error involved in using equation (9a) by considering the approximation

$$
\tau_{+}=\frac{\beta \tau}{1+\beta \mu}
$$

which reproduces both limits $(9 \mathrm{a})$ and $(9 \mathrm{~b})$. This gives

$$
\int_{0}^{\mu_{0}} e^{-\tau+} d \mu=\left(\mu_{0}+\frac{1}{\beta}\right) E_{2}\left(\frac{\tau \beta}{1+\beta \mu_{0}}\right)-\frac{1}{\beta} E_{2}(\tau \beta)=\mu_{0} E_{2}\left(\tau / \mu_{0}\right)\left[1+O\left(\frac{1}{\beta \mu_{0}}\right)\right],
$$


where $\left(\beta \mu_{0}\right)^{-1}<0.05$ for all values $\delta$ used in the observations. Since the expression (15) has qualitatively the same behavior as the more exact expression (8), we may be sure that the error involved in replacing equation (8) by equation (9a) is also of order $\left(\beta \mu_{0}\right)^{-1}$. Thus to a good approximation we obtain

$$
A_{+}=\int_{0}^{\infty} r^{2} \mu_{0} E_{2}\left(\frac{\tau}{\mu_{0}}\right) \Delta B d \tau
$$

We evaluate first the contribution to $A_{+}$from layers that extend no more than 1 " beyond the limb. For these layers $r \approx R$ and $\mu_{0}^{2} \approx 2\left(R-r_{0}\right) / R$; hence equation (17) becomes

$$
A_{+} \approx R^{2} \mu_{0} \int_{0}^{\infty} E_{2}\left(\frac{\tau}{\mu_{0}}\right) \Delta B d \tau
$$

If $\Delta B$ is independent of $\tau$ from $\tau=0$ to $\tau \gg \mu_{0}$, this can be written

$$
A_{+} \approx R^{2} \mu_{0}^{2} \Delta B \int_{0}^{\infty} E_{2}\left(\frac{\tau}{\mu_{0}}\right) \frac{d \tau}{\mu_{0}} \approx R\left(R-r_{0}\right) \Delta B
$$

Here $A_{+}$is linear in $\delta$, the angle subtended by $R-r_{0}$ at the Earth. This is the linear law considered by Dicke and Goldenberg in their analysis of the effects of brightness variation.

On the other hand, consider a situation where $\Delta B=0$ for $\tau>\tau_{0}$, where $\tau_{0} \ll \mu_{0}$ for all values of $\mu_{0}$ used. For the three values of $\delta$ used in the observations, the values of $\mu_{0}$ are $0.12,0.16$, and 0.20 , so we must have $\tau_{0} \ll 0.1$. In this case equation (18) becomes

$$
A_{+} \approx R^{2} \mu_{0} \int_{0}^{\tau_{0}} E_{2}\left(\frac{\tau}{\mu_{0}}\right) \Delta B d \tau \approx \frac{1}{2}(2 R)^{3 / 2}\left(R-r_{0}\right)^{1 / 2} \int_{0}^{\tau_{0}} \Delta B d \tau
$$

since $E_{2} \approx 1$ for small argument. Here we see that $A_{+} \propto \delta^{1 / 2}$. Qualitatively, this $\delta$-dependence arises because the emission from a nearly transparent layer is proportional to its optical thickness along the ray. The optical thickness is $d \tau / \mu \propto d r(R-r)^{-1 / 2}$, and this is integrated from $r=r_{0}$ at the occulting disk to $r=R$ at the limb. The resulting signal amplitude is then proportional to $\left(R-r_{0}\right)^{1 / 2}$, that is, to $\delta^{1 / 2}$.

Finally, we must estimate the contribution from layers well beyond the solar limb. Dicke and Goldenberg used an aperture stop $40^{\prime \prime}$ outside the limb to define the outer limit of the contributing layers. For such a layer at radius $r$ the distance to the limb $(r-R)$ is an appreciable fraction of the distance to the occulting disk $\left(r-r_{0}\right)$. This leads to two changes: first, $\mu_{0}$ varies significantly with respect to $\tau$ in the expression (17) for $A_{+}$; and second, the contribution from the far side $A_{-}$is comparable to that from the near side. From equation (2) we have $\mu_{0} \propto\left(r-r_{0}\right)^{1 / 2}=\left[(r-R)+\left(R-r_{0}\right)\right]^{1 / 2}$, where now the distance $(r-R)$ is comparable to $\left(R-r_{0}\right) \propto \delta$. The quantity $\mu_{0}$ is therefore less sensitive to changes of $\delta$ than in the previous examples where $(r-R) \ll$ $\left(R-r_{0}\right)$. Thus we find that the contribution resulting from brightness variation in the outermost layers resembles that from true oblateness, where the signal is independent of $\delta$.

We may estimate the contribution to $A_{-}$by noting that the limb acts as the occulting disk for the far side. Thus an approximate form for $A_{-}$is simply the right-hand side of equation (17) with $\mu_{0}$ replaced by $[2(r-R) / R]^{1 / 2}$. If $(r-R)<0$, we set $A_{-}=0$. Comparing this with equation (2), we see that the ratio $A_{-} / A_{+}$is approximately $\left[(r-R) /\left(r-r_{0}\right)\right]^{1 / 2}$, which is $\ll 1$ for layers close to the limb, and approaches 1 for layers far from the limb. The contribution $A_{-}$is totally unaffected by the position of the occulting disk, and is therefore independent of $\delta$. This part of the signal is indis- 
tinguishable from true oblateness with the present data, but as Dicke (1970a) has remarked, it is likely to be rather small compared with the observed signal.

\section{COMPARISON WITH THE DATA}

The observations of Dicke and Goldenberg extended over a period of several months. During this period the measured signal varied because of the change in the orientation of the Sun's rotation axis in the frame of the observer. The expected variation with time was computed by Dicke and Goldenberg and is displayed as a solid curve in Figure 1. The lower half of this figure shows the published data plotted separately for the three different values of $\delta$. The amplitude and phase of the solid curve have been adjusted by Dicke and Goldenberg to give the best fit to all the data points, irrespective of $\delta$. A useful check on the fit was that it determines the orientation of the solar axis of rotation. Dicke and Goldenberg concluded that the data did not admit of a linear dependence of signal on $\delta$, and inferred a solar oblateness of $5 \times 10^{-5}$.

We feel that in view of other possible $\delta$-dependences a thorough reexamination is called for. We shall not try to carry one out here, but would simply like to show that a $\delta^{1 / 2}$ law seems to represent the data as well as a $\delta$-independent law. To do this we have scaled the data in the lower portion of Figure 1 by $\delta^{1 / 2} /\left\langle\delta^{1 / 2}\right\rangle$, where $\left\langle\delta^{1 / 2}\right\rangle$ is the arithmetic mean of the three values of $\delta^{1 / 2}$ used in the observations. The scaled data are plotted in the upper half of Figure 1. The amplitude of the solid curve in the lower half of the diagram was varied to minimize the mean square deviation of all the points from the curve, and the optimum amplitude for the upper curve turned out to be nearly the
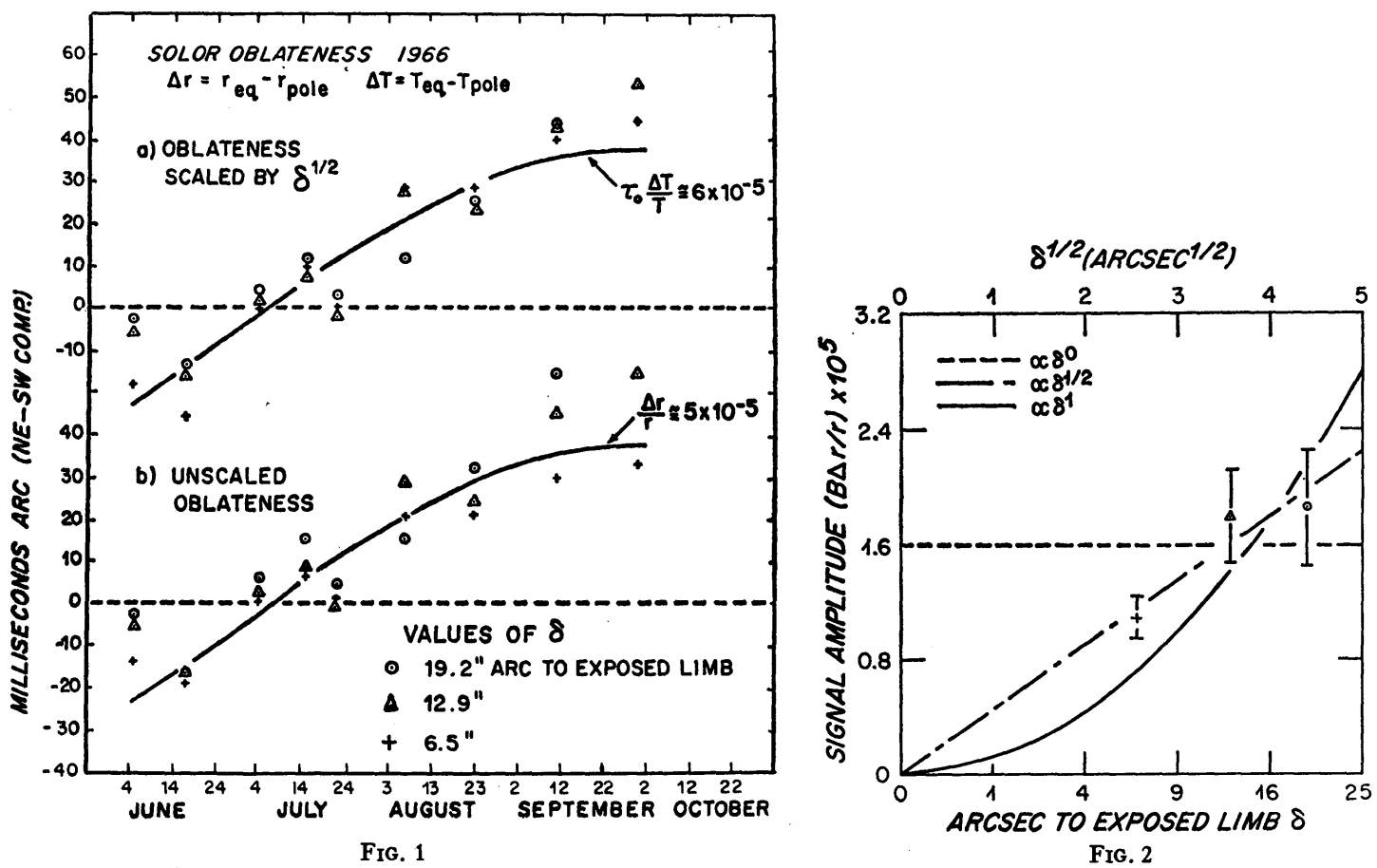

FIG. 1.-Lower portion, published data (Dicke and Goldenberg 1967; Dicke 1970b). Solid curve gives expected time variation for an oblateness of $5 \times 10^{-5}$. Upper portion, data scaled to remove a $\delta^{1 / 2_{-}}$ dependence.

FIG. 2.-Signal amplitude versus $\delta^{1 / 2}$ after correction for surface rotation. Units are $B \Delta r / r$, where $B$ is relative brightness at the occulting disk (Dicke 1970a), and $\Delta r / r$ is measured oblateness (Dicke and Goldenberg 1967). Error bars give the square root of variance for each $\delta$. Curves illustrate three possible dependences on $\delta$. 
same as for the lower curve. The rms deviation of the scaled points from the solid curve was 10 percent less than the rms deviation found from the unscaled points. We conclude that the range of $\delta$ is not sufficient to distinguish whether or not the signal is independent of $\delta^{1 / 2}$.

Processing the data in other ways gives substantially the same result. For example, we subtracted the expected signal associated with surface rotation from the data, and determined the residual amplitude separately for each $\delta$. Each $\delta$-subset of the observations yielded an equivalent residual oblateness $\Delta r / r$, representing that part of the oblateness not associated with surface rotation. Each of these numbers was then multiplied by the relative brightness at the occulting disk $B$ to give a number proportional to signal amplitude. For $\delta=6 " .5,12^{\prime \prime} .9$, and $19^{\prime \prime} .1$, the three values of $B$ are $0.360,0.400$, and 0.432, respectively (Dicke 1970a). The three values of $B \Delta r / r$ are plotted against $\delta^{1 / 2}$ in Figure 2. Error bars show the square root of the variance of $B \Delta r / r$ estimated separately for each $\delta$ from the mean square residuals. The three smooth curves show the relation $B \Delta r / r \propto \delta^{n}$, where $n=0, \frac{1}{2}, 1$. The data are not inconsistent with a $\delta^{1 / 2}$ law, but we feel that additional data are needed before more definite conclusions can be drawn.

In constructing Figures 1 and 2 we have given all the published data equal weight, and have estimated variances in Figure 2 from mean square residuals. However, we have also processed the data using weights based on the error estimates published by Dicke and Goldenberg. This does not lead to a significant improvement in fit, but the inferred values of oblateness are somewhat reduced by this procedure. Finally, we suggest, on the basis of Figure 2, that in future observations of this sort, measurements for $\delta=3^{\prime \prime}$ or $4^{\prime \prime}$ would be quite valuable. The data for larger $\delta$ do not seem as reliable, and might not be as decisive.

We now inquire into the amplitude of the temperature difference $\Delta T$ needed to explain the observations. We equate expression (20) for $A \approx A_{+}$with that part of the signal not explained by surface rotation, namely, $B R \Delta r$, where $\Delta r=28 \mathrm{~km}$; thus,

$$
\frac{1}{B} \int_{0}^{\tau_{0}} \Delta B d \tau=\frac{\Delta r}{\left[2 R\left(R-r_{0}\right)\right]^{1 / 2}} \approx 2.5 \times 10^{-4},
$$

where the value of $r_{0}$ corresponding to $\delta=\left\langle\delta^{1 / 2}\right\rangle^{2}$ has been used. In the special case of a model with $\Delta B=$ const. for $\tau \leq \tau_{0}$ and $\Delta B=0$ for $\tau>\tau_{0}$, this becomes

$$
\tau_{0} \Delta T / T=6 \times 10^{-5} .
$$

We recall the requirement that $\tau_{0} \ll 0.1$ and use $T=5000^{\circ} \mathrm{K}$ to obtain for this model

$$
\Delta T \gg 3^{\circ} \mathrm{K} \text {, }
$$

as a restriction on the temperature difference needed to produce the observed signal. This is not incompatible with the upper bound of $3^{\circ} \mathrm{K}$ found by Dicke and Goldenberg since they did not consider the case $\tau_{0} \ll 0.1$. In particular, if $\tau_{0}=0.01$, a temperature difference from pole to equator of $30^{\circ}$ suffices to explain the observed signal and is consistent with the data.

Of course, it is also possible that the observed signal results from the combined effects of oblateness and temperature variation. In these calculations we have assumed that the various kinds of oblateness signals can be linearly combined. This assumption was already used explicitly when we made the approximation that surfaces of constant $\tau$ are spheres, and it appears to be valid. If a true oblateness $\Delta r / r \sim 10^{-5}$ is present, the preceding formulae for $\tau_{ \pm}, A_{ \pm}$, etc. will be in error by amounts of the order $\Delta r / r, \Delta r /\left(R-r_{0}\right)$, etc., and these are all small. However, there is the possibility that the temperature variation implied by equation (21) will also cause a true oblateness, that is, a distor- 
tion of $\tau$-surfaces relative to gravitational equipotentials; but this effect also appears to be small. In the model considered here the scale height $\lambda$ of the optical depth is approximately a multiple of the density scale height; hence $\lambda \propto T$. Letting $\Delta \lambda$ be the equator-to-pole difference in $\lambda$, we have $\Delta \lambda \approx \lambda \Delta T / T$. With equation (22), this becomes

$$
\Delta \lambda \approx \frac{\lambda}{\tau_{0}} 6 \times 10^{-5} \approx 1 \mathrm{~km}
$$

where we have used $\tau_{0} \approx 0.004$, which applies at the limb. This equation states that at the limb, the radial separation of two $\tau$-surfaces whose mean separation is $\lambda$ changes by about $1 \mathrm{~km}$ from equator to pole. We may assume that the $\tau$-surfaces several scale heights $\lambda$ below the limb coincide with equipotentials (Dicke 1970a), so the maximum displacement of the limb according to equation (24) is 1 or $2 \mathrm{~km}$. This is small compared with $\Delta r \approx 30 \mathrm{~km}$ needed to explain the observations, so the only effect is to modify the estimate of the necessary brightness variation (21) by a few percent. Equation (24) implies that the displacement increases as $\tau_{0}$ decreases, but the brightness of regions beyond the limb also decreases, so the contribution to the signal is still small.

\section{CONCLUDING REMARKS}

The chief conclusion of the previous section is that the observed solar "oblateness signal" can be accounted for by a moderate excess in equatorial temperature as expressed in equation (21). In the example $\Delta T=$ constant for $\tau \leq \tau_{0} \sim 0.01$ and $\Delta T=$ zero otherwise, an equatorial temperature excess of $1^{\circ} \mathrm{K}$ gives a signal comparable to an equatorial radius excess of $1 \mathrm{~km}$. The required temperature excess is then $\sim 30^{\circ}$ and is located in the high photosphere and low chromosphere. It is tempting, therefore, to speculate that the origin of this kind of variation is connected with the mechanical heating of those layers.

It is generally accepted that the chromosphere is heated by waves propagating upward from the photosphere where they are somehow generated in or near the convection zone. The flux of mechanical energy is $\sim \rho w^{2} C$, where $w$ is the rms vertical velocity and $C$ is the local sound speed. Appropriate values of these quantities at $\tau=0.1$ are $\rho=10^{-7} \mathrm{~g} \mathrm{~cm}^{-3}, w=2 \mathrm{~km} \mathrm{sec}^{-1}, C=7 \mathrm{~km} \mathrm{sec}^{-1}$; and we find an energy flux $\sim 2.8 \times$ $10^{9} \mathrm{ergs} \mathrm{cm}^{-2} \mathrm{sec}^{-1}$ (Allen 1963; Roddier 1965; de Jager and Neven 1967). Evaluation of the flux at $\tau=0.01$ gives substantially the same result. The radiant flux is $F_{\odot}=$ $6 \times 10^{10} \mathrm{ergs} \mathrm{cm} \mathrm{cm}^{-2} \mathrm{sec}^{-1}$. Hence the mechanical flux is $4 \times 10^{-2} F_{\odot}$, most of which is dissipated in the chromosphere. The question which we now raise is how much of a variation in this mechanical input with latitude is required to produce the kind of variation implied by equation (21).

Since the layers of interest are optically thin, they radiate per unit area at a rate $4 \pi \int B d \tau$ (where the integration is from 0 to $\tau_{0}$ ). The difference in this emitted radiation from equator to pole is

$$
4 \pi \int_{0}^{\tau_{0}} \Delta B d \tau=4 \pi B 2.5 \times 10^{-4} \approx 4 \times 10^{-4} F \odot
$$

where we have used equation (21), and have set $T=5000^{\circ} \mathrm{K}$ to evaluate $B$. Hence if 1 percent more mechanical energy is dissipated near the equator than near the poles, the observed oblateness signal can be understood. Whether such an asymmetry is to be expected because of rotation or magnetic fields is not known, but the amount of asymmetry required does not seem excessive.

If we postulate the required asymmetry in mechanical flux, we must face the possibility that the associated wave stresses may themselves produce an oblateness. The stress $S_{\mathrm{rr}}$ associated with the mechanical flux is $\rho w^{2}$. As discussed above, this quantity is about $4 \times 10^{3}$ dynes $\mathrm{cm}^{-2}$ at the $\tau=0.1$ level, and it is probably independent of 
altitude in the upper photosphere. A variation with latitude in the magnitude of this stress $\Delta S_{\mathrm{rr}}$ will produce a variation in the weight of overlying material which can be supported, given by $\Delta S_{\mathrm{rr}}=\rho g \Delta r$. Here $\Delta r$ can be interpreted either as the additional thickness of overlying material or as the vertical displacement of a constant- $\tau$ surface. We have seen that the value of $\Delta S_{\mathrm{rr}}$ necessary to produce the required temperature variation $\Delta T$ is $\sim 1 \times 10^{-2} S_{\mathrm{rr}}=40$ dynes $\mathrm{cm}^{-2}$. The density $\rho$ at the solar limb is $\sim 0.2 \times$ $10^{-7} \mathrm{~g} \mathrm{~cm}^{-3}$, whence $\Delta r \sim 0.7 \mathrm{~km}$, which is small. Thus wave heating can produce a $\Delta T$ sufficient to explain the oblateness signal without causing a real oblateness.

In sum, there seem to be at present no physical considerations or observations which can distinguish unambiguously between temperature-induced and oblateness-induced signals. Depending on $\Delta T$, the Sun may be more oblate than the amount proposed by Dicke and Goldenberg, or it may be prolate.

We should like to thank Dr. Rory Thompson for help and advice in the processing of the data.

\section{REFERENCES}

Allen, C W. 1963, Astrophysical Quantities (2d ed.; London: Athlone Press), p 167.

Dicke, R H 1970a, Ap J., 159, 1 1970b, Ann. Rev. Astr and Ap., 8, 30

Dicke, R. H., and Goldenberg, H. M 1967, Phys Rev Letters, 18, 31.

Durney, B. R , and Roxburgh, I. W 1969, Nature, 221, 646

Jager, C. de, and Neven, L. 1967, Solar Phys , 1, 27

Roddier, F 1965, Ann. d'ap , 28, 463. 